Despite the frenzy of research activity, particularly over the past five years, we still know remarkably lítle of the function of the numerous metabolites of vitamin $\mathrm{D}$ or their mode of action on target tissues. The complex interrelationship between vitamin D and the other calcium-regulating hormones and the diverse nature of the organs and tissues involved also emphasises the value of a multidisciplinary approach to the research. This book is particularly valuable not only because it provides an excellent review of the current state of our knowledge of vitamin $\mathrm{D}$ but also because it serves as such a useful reference source of background information for researchers in various areas. A number of the contributors to this volume are leaders of the research groups responsible for the major advances in vitamin D research and the various chapters therefore provide an authoritative and overall dogmatically balanced account of the literature and of current views on the many controversial aspects of vitamin D.

The chemistry and metabolism of the calciferols is reviewed in two chapters by Bell and by Holick and DeLuca respectively. Three subsequent chapters by Norman, Wasserman et al. and by Lawson consider the intestinal system from different aspects. One quarter of the entire text is devoted to two chapters on bone, by Aaron and by Barnes and Lawson, and a large proportion of these chapters consists of a review of the histology and biochemistry of growth cartilage and bone. Establishing the role of vitamin D in bone formation is clearly one of the main priorities for the next decade and the importance attached to the subject in this book is a reflection of this. The physiology and pathology of vitamin $\mathrm{D}$ in man is considered in two more chapters by Stanbury and Mawer, and in the final one Parsons discusses the relationship between it and other calcium-regulating hormones.

Most subiects are covered well but in such a forward-looking book I am surprised that the role of parathyroid and muscle as possible target tissues, and the control of intracellular calcium movement, were not considered in more detail. Overall though this is a valuable contribution to the literature and satisfies a very real need. It will be widely read and appreciated by the large numbers of biochemists, physiologists, nutritionists, clinicians and all others having an interest in calcium and phosphate metabolism.

Ian Dickson

Ian Dickson is a member of the Tissue Physiology Group at the Strangeways Research Laboratory, Cambridge, UK.
Interested in ORIGINS? Alternative!

Keep abreast of new developments in the creation movement by subscribing to the Creation Research Society Quarterly. The following recent research articles in the journal offer important new insights into the problem of origins:

- Post-Fire Regrowth in Relation to
Ecology and Origins

- The Use and Abuse of Astronomy in Dating

- Another Theory of Gravitation

- A Creation Model for Natural Processes

- Amino Acid Racemization in Marine Sediments

- Bristlecone Pines and Tree-RingDating

- The Precision of Nuclear Decay Rates

\section{Centrifugation techniques}

Centrifugation: A Practical Approach. Erited by D. Rickwood. Pp. 224. (Information Retrieval: London and Washington, 1978.) Hardback £8; \$16; paperback $£ 4$; $\$ 9$.

THE subject matter of this book justifies the publishers' claims that it is an introduction to the technique with an essentially practical basis. It discusses differential pelleting, rate zonal and isopycnic methods and their appropriateness for various purposes with different types of macromolecules, subcellular particles and cells. The operation of all types of rotor, including analytical, is described.

There are numerous suggestions for experiments that contribute to the understanding of principles. The comprehensive details provided embrace even the analytical methods to be used after centrifugation. Some of the warnings included imply that the recommended procedures have been modified in the light of experience. Apart from experiments that involve merely the generation or analysis of gradients, expensive equipment is involved. An unsupervised novice would therefore be well advised not to be too ambitious.
On the whole the authors are successful in choosing a happy medium between the risks of overloading the text with qualifying clauses and of presenting oversimplified statements. At some points the grammatical purist may wince, but there are few slips or printers' errors, although "Pedersen" is consistently spelt "Pederson". There is a useful glossary of terms, but symbols are not invariably standard throughout the book.

With two exceptions the number of references at the end of each chapter has a maximum of ten. This restraint is commendable in a book of this nature, but occasionally results in a misleading impression. For instance, the short section dealing with the hydrostatic pressure of gradients contains only recent references and bears no hint that the existence of pressure gradients in any solution being centrifuged has long been recognised.

As there is a paucity of publications covering the same field at an elementary level, this book should find wide acceptance, especially as the price of the paperback should encourage younger workers to have a personal copy.

P. A. Charlwood

P. A. Charlwood is on the scientific staff of the $M R C$ at the National Institute for Medical Research, London, UK.
- On Methods of Teaching Origins: A Progress Report

- Phylogenetic Development of Adipose Tissue

- Galaxy Clusters and the Mass Anomaly

- Radiohalos in Coalified Wood

- Fossil Zones

- Which Animals do Predators Really Eat?

- Probability and Missing Transitional Forms

If you are interested in alternative solutions to the problem of origins, the Creation Research Society invites you to subscribe to the CRS quarterly - over 200 pages per year of large format journal - $\$ 13.00$ year ( $\$ 14.00$ foreign). Checks only, please. Send to Subscription secretary, CRS, 2717 Cranbrook Road, Ann Arbor, MI 48104. For more information write CRS, 27117 Langside Ave., Canyon Country, CA 91351.

Circle No. 18 on Reader Enquiry Card. 